\title{
Bouvard et Pécuchet : croyances et savoirs
}

\section{Gisèle Séginger}

\section{(2) OpenEdition}

\section{Journals}

Édition électronique

URL : http://journals.openedition.org/aes/549

DOI : $10.4000 /$ aes.549

ISSN : 2258-093X

\section{Éditeur}

Laboratoire LISAA

\section{Référence électronique}

Gisèle Séginger, « Bouvard et Pécuchet: croyances et savoirs », Arts et Savoirs [En ligne], 1 | 2012, mis en ligne le 15 février 2012, consulté le 03 mai 2019. URL : http://journals.openedition.org/aes/549 ; DOI : 10.4000/aes.549

Ce document a été généré automatiquement le 3 mai 2019.

Centre de recherche LISAA (Littératures SAvoirs et Arts) 


\title{
Bouvard et Pécuchet : croyances et savoirs
}

\author{
Gisèle Séginger
}

1 S'attaquant aux superstitions et à la religion, la Révolution française a stimulé l'essor scientifique du XVIII ${ }^{\mathrm{e}}$ siècle. Au XIX ${ }^{\mathrm{e}}$ siècle, les recherches se diversifient ainsi que l'enseignement; les lieux de savoirs et les revues se multiplient ${ }^{1}$. Des verrous épistémologiques sautent, des tabous moraux et religieux tombent. Même l'origine du monde et de la vie, l'homme et les religions sont entraînés sous les lumières de la science. Positivisme, objectivité, observation, expérience sont les maîtres mots de l'esprit scientifique en pleine expansion. Les sciences de la matière et du vivant fournissent des modèles d'intelligibilité aux sciences de l'homme naissantes. Flaubert partage avec son époque une fascination pour la positivité des sciences, et il voudrait que l'on étudiât l'homme sans préjugés moraux ni religieux, avec des méthodes de naturaliste (lettre à L. Colet du 31 mars 1853). Il écrit Bouvard et Pécuchet (histoire des années 1840-1850) à un moment (1872-1880) où, malgré le développement d'une piété mariale, de dévotions populaires, la mise à l'Index de nombreux ouvrages, la religion mène un combat d'arrièregarde contre l'esprit moderne. Elle garde une influence dans la société et les mœurs, surtout dans les milieux conservateurs - Bouvard et Pécuchet le montre - mais le prêtre est désormais confronté à une figure du savoir, celle du médecin (Vaucorbeil dans le roman), aussi prestigieuse sinon plus. L'aura des sciences vient de ce qu'elles s'occupent du vrai et les penseurs positivistes (Littré, Maury, Taine, Renan), dont Flaubert recommande la lecture à ses correspondants ${ }^{2}$, servent de relais à une diffusion de l'esprit scientifique auprès d'un public lettré. Comment comprendre alors la rédaction d'un roman qui s'en prend aux savoirs? Après avoir vanté les mérites de la science dans les années 1850-1860 et l'avoir érigée en modèle d'impersonnalité pour la littérature, Flaubert en viendrait-il à prendre ses distances avec l'esprit scientifique?

2 Une relecture du roman dans le contexte de l'œuvre entière et la confrontation de certains énoncés avec les déclarations de la Correspondance nous permettront de nuancer la réflexion de Flaubert sur les sciences. Bouvard et Pécuchet n'est pas la première œuvre qui suggère ses réticences à l'égard d'une certaine approche de la science. Dans ce 
roman, ce n'est d'ailleurs pas tant la recherche scientifique qui est mise en cause qu'une conception du vrai et l'illusion qu'on peut échapper à tout conditionnement (culturel, moral, idéologique, psychologique), atteindre des certitudes; c'est la volonté de plier le monde à notre désir et à notre besoin. Bouvard et Pécuchet veulent arrêter la recherche, obtenir le dernier mot de la science, fixer en dogmes les découvertes, transformer les lois en mode d'emploi pour agir sur le monde, et s'emparer du pouvoir créateur. Le savoir se rapproche de la croyance. Contre cette confusion, Flaubert emploie une ironie qui déconstruit les savoirs les uns après les autres, mais qui est aussi paradoxalement un nouveau moyen de connaissance. Fiction des savoirs qui en libère les potentialités imaginaires et narratives (les savoirs contiennent implicitement des récits sur le monde), Bouvard et Pécuchet ne rompt pas avec toute visée philosophique et épistémologique. En effet, cette fiction a une portée métathéorique et méta-scientifique.

\section{Positivisme critique et religion positiviste}

Dans les années où il prépare Madame Bovary (1851-1856), Flaubert s'est éloigné de la littérature romantique qui l'avait fasciné pendant sa jeunesse. En 1852, il réfléchit sur ce que doit être le roman moderne par rapport au roman idéaliste et sentimental de Lamartine, Graziella: «Plus il ira, plus l'Art sera scientifique [...]» $»^{3}$. Contre les édulcorations romantiques et les mollesses de la phrase, symptômes de l'implication excessive de la personnalité, il imagine « un style [...] qui serait rythmé comme le vers, précis comme le langage des sciences $»^{4}$. Dans les années Bovary, Flaubert donne souvent en exemple l'attitude scientifique. En 1853, son désir de positivisme est tel qu'il en vient à imaginer une science naturelle de l'homme :

Il y a ainsi une foule de sujets qui m'embêtent également par n'importe quel bout on les prend. (C'est qu'il ne faut pas sans doute prendre une idée par un bout, mais par son milieu). Ainsi Voltaire, le magnétisme, Napoléon, la révolution, le catholicisme, etc., qu'on en dise du bien ou du mal, j'en suis mêmement irrité. La conclusion, la plupart du temps, me semble acte de bêtise. C'est là ce qu'ont de beau les sciences naturelles: elles ne veulent rien prouver. Aussi quelle largeur de faits et quelle immensité pour la pensée! Il faut traiter les hommes comme des mastodontes et des crocodiles. Est-ce qu'on s'emporte à propos de la corne des uns et de la mâchoire des autres? Montrez-les, empaillez-les, bocalisez-les, voilà tout ; mais les apprécier, non. Et qui êtes-vous donc vous-mêmes, petits crapauds ?5

Certes, du point de vue épistémologique, il méconnaît la spécificité des sciences de l'homme. Mais l'essentiel n'est pas là. Flaubert n'a pas la prétention d'être un historien des sciences, un scientifique, ni même un philosophe. Le rapprochement d'ordre purement métaphorique n'a d'autre fonction que de l'aider à penser une nouvelle forme romanesque. Quelques jours après cette lettre, on le voit bien lorsqu'il définit ainsi l'objectif de la littérature : elle " prendra de plus en plus les allures de la science; elle sera surtout exposante, ce qui ne veut pas dire didactique. Il faut faire des tableaux, montrer la nature telle qu'elle est, mais des tableaux complets, peindre le dessous et le dessus. $»^{6}$

Tout en rédigeant Madame Bovary - qui montre quelques méfaits de l'idéalisme romantique - Flaubert réfléchit sur l'évolution du genre romanesque et de la littérature qu'il faut débarrasser de la chlorose lamartinienne. Il corrige dans le même temps les poèmes de Louise Colet souvent trop sentimentaux et accueillants pour les idées et préjugés du temps. C'est dans ce contexte, qu'il définit l'exposition - dont le modèle est scientifique - comme une poétique efficace pour éviter le pathos, les préjugés et les 
jugements. Le roman doit être une machine qui déjoue le désir du savoir. La science valorisée par Flaubert n'est donc pas celle de Homais ou de Bouvard et Pécuchet. Une note de la fin des années 1859 indique assez clairement la qualité essentielle que l'écrivain retient de la méthode scientifique: «La [Science] < Critique > est la dixième Muse $»^{7}$. Il a raturé le mot "Science» (avec une majuscule) et l'a remplacé par "Critique». On comprend donc ce qui l'intéresse: une méthode et non des savoirs particuliers, l'interrogation et le doute et non la volonté de savoir et la recherche de la vérité. La correction du mot "Science » en "Critique " nous laisse deviner comme une réticence à l'égard de la science lorsque les savoirs qu'elle produit contredisent sa dimension critique et arrêtent sa dynamique. Or, c'est avant tout sa puissance critique que Flaubert retient de la science lorsqu'il l'érige en modèle pour la littérature. Aussi le voit-on adapter au domaine de l'esthétique et de la poétique narrative les deux procédures qui caractérisent la méthode scientifique (et non pas les sciences en tant que productrices de savoirs): le roman sera exposant et critique. Flaubert attaque la littérature " probante $»^{8}$ qui porte des jugements sur le monde, et se rend coupable de cette bêtise suprême qu'est pour Flaubert le désir de conclure.

Or, il est intéressant de noter qu'il affirme pour la première fois ce dégoût des jugements assertifs au sujet d'un philosophe qui pervertit le positivisme au point d'en faire un nouveau dogme: Auguste Comte considère l'âge positiviste comme le dernier stade de l'humanité, son aboutissement. Flaubert admire la méthode positiviste non la philosophie positiviste dans laquelle il détecte une dérive. En 1850, il qualifie l'Essai de philosophie positive de Comte de livre socialiste, car il perçoit une utopie de l'humanité en gloire, et le retour d'une croyance : « Il y a là-dedans des mines de comique immenses, des Californies de grotesque. ${ }^{9}$ Comte trace les progrès de l'esprit de la philosophie théologique, puis métaphysique jusqu'à la philosophie positive. Celle-ci marquerait la fin de l'Histoire. Il reconnaît que la recherche des causes est vouée à l'échec, mais il n'échappe pas à la critique flaubertienne lorsqu'il déplace la certitude dans l'ordre des phénomènes, se montrant convaincu qu'ils sont tous «assujettis à des lois naturelles invariables, dont la découverte précise et la réduction au moindre nombre possible sont le but de tous nos efforts $»^{10}$. De même, rejetant la religion à cause de ses croyances archaïques, Bouvard et Pécuchet croient cependant à la possibilité de comprendre et de maîtriser les phénomènes par quelques lois bien établies. C'est ce travers d'une époque qui change de religion ${ }^{11}$ que Flaubert met en scène de manière critique dans son roman. La croyance descend du ciel sur la terre mais n'en demeure pas moins structurellement une croyance illusoire. Le Cours de philosophie positive suscite ce commentaire : «L'ineptie consiste à vouloir conclure ${ }^{12}$. La métaphysique fait retour dans ce positivisme dégénéré. Il y a donc pour Flaubert positivisme et positivisme : il y a Littré, Maury et Renan qu'il admire, et d'un tout autre bord, sur le versant d'une nouvelle métaphysique, le ridicule Auguste Comte. Pendant la préparation de Bouvard et Pécuchet, Flaubert a consulté le livre de Ludwig Büchner, Science et nature (traduit en 1866), qui comportait un chapitre intitulé «Les positivistes ou une nouvelle religion $»^{13}$. L'ouvrage résume les idées des adeptes d'Auguste Comte. Il insiste donc sur la substitution de l'humanité à Dieu et la foi de l'homme en luimême qui avait fait dire à Flaubert: «L'adoration de l'humanité pour elle-même et par elle-même [...] engendre du vent $»^{14}$. Foi dans la Science, foi en l'homme, les deux vont de pair, et suscitent une égale moquerie de la part de Flaubert. 
7 Pourtant, il arrive au moins une fois, où dans sa Correspondance, se laissant probablement emporter par sa haine de la morale, Flaubert en vient à rêver ce curieux avenir des sciences de l'homme :

Les matérialistes et les spiritualistes empêchent également de connaître la matière et l'esprit, parce qu'ils scindent l'un de l'autre. Les uns font de l'homme un ange et les autres un porc. Mais avant d'en arriver à ces sciences-là (qui seront des sciences), avant d'étudier bien l'homme, n'y a-t-il pas à étudier ses produits, à connaître les effets pour remonter à la cause ? Qui est-ce qui a, jusqu'à présent, fait de l'histoire en naturaliste? A-t-on classé les instincts de l'humanité et vu comment, sous telle latitude, ils se sont développés et doivent se développer? Qui est-ce qui a établi scientifiquement comment, pour tel besoin de l'esprit, telle forme doit apparaître, et suivi cette forme partout, dans les divers règnes humains? Qui est-ce qui a généralisé les religions ? Geoffroy Saint-Hilaire a dit : le crâne est une vertèbre aplatie. Qui est-ce qui a prouvé, par exemple, que la religion est une philosophie devenue art, et que la cervelle qui bat dedans, à savoir la superstition, le sentiment religieux en soi, est de même matière partout, malgré ses différences extérieures, correspond aux mêmes besoins, répond aux mêmes fibres, meurt par les mêmes accidents, etc. ? Si bien qu'un Cuvier de la Pensée n'aurait qu'à retrouver plus tard un vers ou une paire de bottes pour reconstituer toute une société et que, les lois en étant données, on pourrait prédire à jour fixe, à heure fixe, comme on fait pour les planètes, le retour des mêmes apparitions. Et l'on dirait : nous aurons dans cent ans un Shakespeare, dans vingt-cinq ans telle architecture. Pourquoi les peuples qui n'ont pas de soleil ont-ils des littératures mal faites? Pourquoi y a-t-il, et y a-t-il toujours eu, des harems en Orient, etc. ?

On a beaucoup battu la campagne sur tout cela, on a été plus ou moins ingénieux ; mais la base a toujours manqué. La première pierre est à trouver. La critique des œuvres de la Pensée a toujours été faite à un point de vue étroit, rhéteur, et la critique de l'histoire faite à un point de vue politique, moral, religieux, tandis qu'il faudrait se placer au-dessus de tout cela, dès le premier pas. Mais on a eu des sympathies, des haines; puis l'imagination s'en est mêlée, la phrase, l'amour des descriptions et enfin la rage de vouloir prouver, l'orgueil de vouloir mesurer l'infini et d'en donner une solution. Si les sciences morales avaient, comme les mathématiques, deux ou trois lois primordiales à leur disposition, elles pourraient marcher de l'avant. Mais elles tâtonnent dans les ténèbres, se heurtent à des contingents et veulent les ériger en principes. Ce mot, l'âme, a fait dire presque autant de bêtises qu'il y a d'âmes! Quelle découverte ce serait par exemple qu'un axiome comme celui-ci : tel peuple étant donné, la vertu y est à la force comme trois est à quatre ; donc tant que vous en serez là vous n'irez pas là. ${ }^{15}$

8 Toutefois, aussitôt se reprend-il, et il remet à sa juste place cette rêverie digne de Bouvard et Pécuchet: "Il est bien tard, je déraisonne passablement [...]». Simplifier, totaliser, maîtriser, ces trois opérations caractériseront le rationalisme déterministe de Bouvard et Pécuchet.

\section{De La Tentation de saint Antoine à Bouvard et Pécuchet}

Flaubert considérait Bouvard et Pécuchet comme «testament " ${ }^{16}$. De fait, c'est un aboutissement et non pas un apax dans son œuvre, une subite révolution littéraire qui à elle seule mériterait de sacrer Flaubert comme le grand Patron de la modernité. Certes ce roman fait de théories et de savoirs touche aux limites du genre romanesque. Il entraîne dans le broyeur de la critique tous les discours et parfois même ceux de son auteur. Toutefois la critique du savoir scientifique n'est pas nouvelle dans l'œuvre flaubertienne 
bien qu'elle soit plus systématique dans le dernier roman dont elle fait éclater le cadre romanesque traditionnel.

Flaubert considérait Bouvard et Pécuchet comme le pendant de La Tentation de saint Antoine dont il avait publié la dernière version en $1874^{17}$, vingt-cinq ans après en avoir achevé une première version en 1849. Or, cette œuvre exposait la renaissance infinie de l'illusion religieuse. Parmi les figures tentatrices figurait une allégorie de la Science tandis que le texte publié en 1874 terminait la série des apparitions religieuses par une vision scientifique : la naissance de la vie sous la forme d'être microscopique imaginé à partir des théories de l'époque ${ }^{18}$. L'œuvre de 1874 mettait ainsi en scène un rapport entre croyances et savoirs qui serait le sujet de l'œuvre suivante.

11 Dans La Tentation de 1849, le Diable se présente aux Péchés comme le créateur de « l'éternelle illusion » qui pousse les hommes vers les désirs. D’une part, il fait apparaître aux yeux du saint halluciné un Sphinx mélancolique - il a perdu le savoir et n'a plus aucun secret à dévoiler - qui veut s'envoler sur le dos de la Chimère, de l'Illusion ; d'autre part il met en scène une allégorie de la Science, dévorée d'un désir de connaissance qui la torture, comme plus tard Pécuchet sera animé d'un «besoin de vérité » qui deviendra « une soif ardente » (310). Figure mélancolique comme le Sphinx, la Science déplore son sort :

J'ai des envies, je voudrais faire quelque chose, et des profondeurs de moi-même tirer une création nouvelle. Si je pouvais pénétrer la matière, embrasser l'idée, suivre la vie dans ses métamorphoses, comprendre l'Être dans tous ses modes et de l'un à l'autre remontant ainsi les causes, comme les marches d'un escalier, réunir à moi ces phénomènes épars et les remettre en mouvement dans la synthèse d'où les a détachés mon scalpel... peut-être alors que je ferais des mondes... ${ }^{19}$

Cette Science prométhéenne veut le Savoir pour s'emparer du pouvoir de créer, rivalisant ainsi avec Dieu. L'écriture métaphorique donne au désir totalisateur la forme d'une ascension, et elle ébauche cette figure bien flaubertienne de perfection et de maitrise ${ }^{20}$ qu'est la pyramide, figure aussi de la bêtise et du bourgeois amateur de certitudes ${ }^{21}$. Bien des personnages à idées fixes - Sénécal dans L'Éducation sentimentale et Pécuchet - auront un trait physiognomonique commun : un crâne en pointe.

Figure allégorique du désir de certitude, la Science de 1849 se rapproche finalement de l'allégorie de la Foi : «Ah! si la vérité t'est connue, tends-moi la main pour me faire gravir jusqu'à elle, satisfais ma raison avec ta croyance, rassasie mon âme avec ton Dieu car c'est vers la cause aussi que j'aspire [...]». Toutefois, elle finit par réaffirmer sa différence : la Foi interdit, blâme tandis que pour sa part elle accepte tout, étudie tout; elle est plus large, synthétique, et sans préjugés moraux :

[...] tu te retires des intelligences les plus hautes, moi je descends tout entière dans les petitesses les plus infimes et j'observe des mondes dont tu ne soupçonnes pas l'existence. Mais un temps viendra où les choses seront lavées des malédictions dont tu les couvres, ce qui est obscur resplendira, ce qui est informe se complétera, ce qui semble monstrueux apparaîtra superbe ; j'expliquerai le corps comme l'âme, la matière comme l'esprit, le péché comme la pénitence, le crime comme la vertu, le mal comme le bien, et je rajeunirai sans cesse tandis que tu te courberas vers la décrépitude ${ }^{22}$.

14 Le rêve de substituer la Science à la Religion caractérise le positivisme de la fin des années 1840 dont on trouve un témoignage dans L'Avenir de la science: pensées de $1848^{23}$. Renan avait écrit ce livre en 1848 et il se terminait par un chapitre intitulé « Foi à la science». Mais il ne l'a publié qu'en 1890 - sans rien y changer - pour porter un témoignage sur 
l'état d'esprit d'une époque révolue, par rapport auquel l'homme circonspect (et dont on a souvent critiqué le fin scepticisme) avoue avoir bien pris ses distances.

De la première apparition de la Science dans l'œuvre fictionnelle du jeune Flaubert en 1849 , retenons encore qu'elle fait partie des tentations dangereuses (et non du groupe des Vertus), aux côtés de l'Orgueil (dont elle est l'enfant), de la Luxure, de la Logique... Savoir pour agir sur le réel est la tentation récurrente de tous les personnages qui souffrent d'une libido sciendi dans l'œuvre flaubertienne. La volonté de savoir et de conclure cache une volonté de pouvoir - qu'incarne à nouveau Homais dans Madame Bovary. La cible de Flaubert n'est donc pas la science mais ceux qui veulent en faire un nouvel ordre et les éventuelles dérives sociales qui en découlent. Il ne s'en prend pas aux savants qui cherchent, mais aux utilisateurs qui demandent du savoir prêt-à-utiliser. Dans sa Correspondance il fait bien la différence et il attaque les vulgarisateurs, «les gens de goût, les gens à enjolivements, à purifications, à illusions, ceux qui font des manuels d'anatomie pour les dames, de la science à la portée de tous [...] »" Le mot important pour comprendre Bouvard et Pécuchet - illusion - est déjà là, dans cette lettre de 1853.

Madame Bovary est un roman des illusions qu'elles soient amoureuses ou scientifiques. Dans les deux cas - qu'il s'agisse d'Emma ou de Homais - les personnages veulent faire violence au réel. Homais défend un positivisme scientifique aussi intransigeant que celui de Bouvard et Pécuchet. Il croit aux pouvoirs de la science. Dans Madame Bovary, Flaubert confronte une première fois les livres et le réel, lorsque le Pharmacien pousse Charles Bovary à expérimenter le savoir des livres en opérant Hippolyte. Il monte une mise en scène théâtrale dont on trouvera beaucoup d'exemples dans le dernier roman : «C'était M. Homais qui avait organisé dès le matin tous ces préparatifs, autant pour éblouir la multitude que pour s'illusionner lui-même. ${ }^{25}$ Et le pharmacien semble incapable de désillusion. La Science de 1849 voulait faire des mondes, Homais veut redresser la nature. L'échec du pied-bot ne lui sert pas de leçon, et à la fin du roman il veut encore guérir un aveugle. Un tel miracle avait été réussi par Jésus avec l'aveugle de Jéricho grâce à la foi (Évangile de Marc, X, 46-53). Mais la science reste impuissante. Le Pharmacien n'a plus qu'à manipuler l'Opinion pour que soit enfermé cet être rebelle qui défie son pouvoir, et l'ordre social par son vagabondage. La dynamique n'est pas totalement différente dans le cas d'Emma malgré sa mort et ce sera encore celle de Bouvard et Pécuchet. Emma est désillusionnée de la vie hors du couvent, puis du mariage, puis d'un amant, puis d'un autre, et elle se laisse reprendre toujours par le rêve de l'idéal, jusqu'à la fin où n'ayant pas trouvé l'Amant parfait, elle baise avec passion le corps du Christ sur le crucifix, révélant ainsi la nature de l'illusion comme phénomène de croyance. Bouvard et Pécuchet déplace à Chavignolles un travers de l'héroïne d'Yonville. La poursuite de l'idéal et de l'Amour se transmute en désir de savoir et en quête de vérité. Il y a un bovarysme de la connaissance qui s'alimente au même mécanisme de l'illusion et de l'aspiration que la quête d'amour, ou le désir de croire. Flaubert crée dans le dernier roman un rythme à deux temps, avec l'élan et la retombée, comme après l'échec des conserves ( $\mathrm{La}$ désillusion fut complète. », 115) ou après l'étude de la grammaire ("Ils en conclurent que la syntaxe est une fantaisie et la grammaire une illusion. », 218).

Dans La Tentation de saint Antoine, le Diable emportait saint Antoine (l'homme de la Croyance) sur ses cornes au-dessus de l'univers, dans un espace sans bornes pour remettre en question toutes ses certitudes. Confrontées à l'infini, ses idées tombaient les unes après les autres comme autant d'illusions liées à un point de vue d'abord trop limité. Le Diable se faisait l'avocat d'un relativisme généralisé, d'un scepticisme auquel aucune 
théorie philosophique ne résistait. Utilisant d'abord des arguments kantiens pour invalider la capacité de l'esprit à atteindre autre chose que des phénomènes formatés par l'esprit humain, le Diable finissait par émettre l'hypothèse d'une Illusion généralisée, le monde n'étant qu'une représentation de l'esprit, et l'existence de celui-ci devenant à son tour douteuse :

[...] si tout cela n'était que dérision infinie, qu'il n'y eût que néant? Ah! Tu ne conçois pas que le néant puisse être! Mais qui te dit que ce n'est pas l'absurde au contraire qui est le vrai, qu'il y ait même quelque chose de vrai ? On ne prouve rien et quand même on prouverait tout, jamais une preuve n'existe que par rapport au monde qu'elle concerne et à l'intelligence qui la perçoit et si ce monde lui-même n'est pas, si cet esprit n'est pas?

Deux notes des manuscrits éclairent ce passage, la première établit cette équivalence : «Nihilisme, idéalisme» (N.a.fr. 23671, fo $180 \mathrm{r}^{\circ}$ ). La seconde dans le premier scénario partiel de l'épisode du vol dans l'espace élabore l'argument final du Diable : « Si tout cela n'était qu'une illusion, s'il n'y avait rien du tout? fantasmatisme » ( $f^{\circ} 186 \mathrm{r}^{\circ}$, N.a.fr. 23671).

19 Cet argument fort - à l'encontre de la possibilité d'une connaissance - se retrouve encore dans La Tentation de saint Antoine de 1874. Dans cette œuvre très différente du texte de $1849^{26}$, le Diable n'apparaît sous sa forme médiévale que dans la sixième partie. On découvre alors qu'il avait pris l'apparence d'Hilarion, le disciple du saint, qui avait aussi remplacé les allégories des Péchés et la Logique. Passé maître dans la controverse, Hilarion attaque les certitudes religieuses du saint par divers arguments, et après lui avoir révélé que toutes les religions sont mortelles et que son Dieu passera comme les autres, il dévoile ainsi son identité : «Mon royaume est de la dimension de l'univers; et mon désir n'a pas de bornes. Je vais toujours, affranchissant l'esprit et pesant les mondes, sans haine, sans peur, sans pitié, sans amour, et sans Dieu. On m'appelle la Science $»^{27}$. La critique - pourtant bien différente de la totalisation qu'incarnait la Science en 1849 - est attirée dans la liste des tentations à côté des diverses croyances, comme si toute pensée était menacée de sclérose. Puis, se transformant en Diable comme pour confirmer cette hypothèse, Hilarion emportait Antoine sur ses cornes. Et le voyage philosophique s'achevait sur un argument similaire à celui de la première Tentation :

Peut-être que le monde étant un flux perpétuel des choses, l'apparence au contraire ne soit tout ce qu'il y a de vrai, l'illusion la seule réalité.

Mais es-tu sûr de voir ? Es-tu même sûr de vivre ? Peut-être qu'il n'y a rien !28

Flaubert s'est documenté en 1846 sur le bouddhisme ${ }^{29}$ et la théorie indienne de la maya (ou illusion universelle ${ }^{30}$ ) qui lui sert probablement pour imaginer la conclusion du Diable dès 1849 et à nouveau dans l'œuvre de 1874. Ce n'est que plus tard qu'il lira Schopenhauer et y trouvera en 1879 quelques idées qui font écho aux siennes : «Idéaliste et pessimiste, ou plutôt bouddhiste. - Ça me va. $»^{31}$ Dans Bouvard et Pécuchet, dont l'action est située dans les années 1840 et 1850 (alors que la vogue de Schopenhauer en France est plus tardive), c'est la lecture de Hegel qui pousse les personnages dans la direction d'un idéalisme nihiliste : «La certitude que rien n'existe (si déplorable qu'elle soit) n'en est pas moins une certitude. Peu de gens sont capables de l'avoir. Cette transcendance leur inspira de l'orgueil » (316). Mais là où le Diable posait une question, émettait une hypothèse Bouvard et Pécuchet ont une "certitude». Le doute radical de Bouvard et Pécuchet se métamorphose en nihilisme : ils font de la négation une nouvelle affirmation; la critique poussée à l'extrême se retourne en conclusion. 


\title{
L'infini et les savoirs
}

21 La Science-Croyance (l'allégorie de 1849) et la Science critique (Hilarion) ont toutes deux une descendance dans Bouvard et Pécuchet, les deux personnages faisant tour à tour l'expérience de ces deux perspectives. Les réflexions de la Correspondance sur l'infini qui nous déborde et sur l'univers insaisissable se retrouvent dans la réplique du Diable de 1874 lorsque - après avoir imaginé l'hypothèse de l'idéalisme (le réel n'est qu'une représentation ${ }^{32}$ ) - il évoque la labilité du monde : «À moins que le monde étant un flux perpétuel des choses, l'apparence au contraire ne soit tout ce qu'il y a de plus vrai, l'illusion la seule réalité. ${ }^{33}$ Plus de certitude donc, ni de réel par-delà nos représentations, mais seulement un flux d'apparences changeantes. Malgré l'amour des certitudes, il est dans Bouvard et Pécuchet quelques brefs moments de doute similaires (bien vite surmontés par un regain du désir de savoir) comme ce passage où Bouvard joue le rôle du Diable auprès d'un Pécuchet toujours plus obstiné que lui dans la recherche de la vérité : «[...] tout passe, tout coule. La création est faite d'une matière ondoyante et fugace. Mieux vaudrait nous occuper d'autre chose!» (150). De La Tentation à Bouvard et Pécuchet, l'insaisissable, l'ondoyant et le fugace déjouent le désir de croire. Impossible alors de tenir un discours car celui-ci a besoin d'articulations (cause, conséquence) et de limites, une cause et un but. En 1857, Flaubert essayait de guérir Mlle Leroyer de Chantepie de sa mélancolie religieuse en lui prêchant l'acceptation de l'illimité et de l'insaisissable :

\begin{abstract}
Le but, la cause! Mais nous serions Dieu, si nous tenions la cause, et à mesure que nous irions, elle se reculera indéfiniment, parce que notre horizon s'élargira. Plus les télescopes seront parfaits et plus les étoiles seront nombreuses. Nous sommes condamnés à rouler dans les ténèbres et dans les larmes.

Quand je regarde une des petites étoiles de la Voie Lactée, je me dis que la Terre n'est pas plus grande que l'une de ces étincelles. Et moi qui gravite une minute sur cette étincelle, qui suis-je donc, que sommes-nous ? Ce sentiment de mon infirmité, de mon néant, me rassure. Il me semble être devenu un grain de poussière perdu dans l'espace, et pourtant je fais partie de cette grandeur illimitée qui m'enveloppe. Je n'ai jamais compris que cela fût désespérant, car il se pourrait bien qu'il n'y eût rien du tout derrière le rideau noir. L'infini, d'ailleurs, submerge toutes nos conceptions et, du moment qu'il est, pourquoi y aurait-il un but à une chose aussi relative que nous ? ${ }^{34}$
\end{abstract}

La question de Pécuchet («Quel est le but de tout cela?») et l'hypothèse de Bouvard («Peut-être qu'il n'y a pas de but?»,139) se font l'écho des réflexions de l'écrit privé.

Peut-on dire alors que Flaubert intègre parfois son point de vue dans Bouvard et Pécuchet et que rien n'échappe au jeu de massacre, pas même la position de l'auteur? On l'a souvent écrit, surtout à propos de ce passage :

Alors une faculté pitoyable se développa dans leur esprit, celle de voir la bêtise et de ne plus la tolérer.

Des choses insignifiantes les attristaient: les réclames des journaux, le profil d'un bourgeois, une sotte réflexion entendue par hasard. (319)

Flaubert contre Flaubert? Ce n'est pas sûr. Certes, dans la Correspondance, Flaubert se laisse lui-même aller à des moments d'indignation ${ }^{35}$ de plus en plus nombreux dans les années 1870-1880, probablement à cause des deuils successifs, des désastres nationaux et enfin des problèmes financiers qui l'affectent profondément par ailleurs. En 1880, tandis qu'il rédige le "dernier chapitre», il s'écrie: "L'insupportabilité [sic] de la sottise 
humaine est devenue chez moi une maladie, et le mot est faible. Presque tous les humains ont le don de m'exaspérer et je ne respire librement que dans le désert. $»^{36}$ Bouvard et Pécuchet attire dans le cercle de la Bêtise un refus qui semble bien être celui de l'auteur lui-même. Effet de miroir ou logique du repoussoir?

Bouvard et Pécuchet ne tolèrent plus, s'attristent, et sont entièrement animés par des passions réactives. Or, l'attitude de Flaubert se voulait différente même si les années noires le voient faiblir - à regret - dans ses bonnes résolutions. En effet, l'artiste - selon Flaubert - se devait de surplomber le monde, détaché et serein, contrairement aux autres bourgeois. Je cite une lettre significative à cet égard qui date de 1845, c'est-à-dire d'un moment où, abandonnant l'exaltation romantique, il construisait dans L'Éducation sentimentale de 1845 une figure de l'artiste impersonnel ${ }^{37}$ : "À force de vouloir tout comprendre, tout me fait rêver. Il me semble pourtant que cet ébahissement-là n'est pas de la bêtise. Le bourgeois par exemple est pour moi quelque chose d'infini. Tu ne peux pas t'imaginer $"^{38}$. L'activité de l'écrivain qui rêve et écrit en exposant n'est pas la même que celle de ces demi-critiques que sont Bouvard et Pécuchet et qui concluent toujours d'une manière ou d'une autre, qui jugent et établissent des partages : vrai/faux, bon/mauvais, bien/mal, bête/intelligent. Exposer est une tout autre activité. Je reviens sur la note du Carnet 2, déjà citée mais dont la suite peut maintenant s'éclairer: «La [Science] < Critique > est la dixième Muse et la Bonté la quatrième Grâce ». Il ne faut pas entendre le mot «Bonté » en un sens moral mais esthétique comme l'indique le mot "Grâce ». Au nombre de trois dans la mythologie (Aglaé, Thalie, Euphrosyne), les Grâces étaient des divinités festives, qui incarnaient les dons de la vie (art, beauté, joie, sagesse, douceur) et elles étaient les compagnes de Vénus. La quatrième Muse inventée par Flaubert - Bonté est une muse de la synthèse et de l'acceptation, propice à l'art. En 1855, tandis qu'il élaborait son premier roman dialogique et égalisateur - Madame Bovary - qui atténuait les différences entre le mari et l'amant, le prêtre et le défenseur de la science, il confiait à Louis Bouilhet :

Que je sois pendu si je porte jamais un jugement sur qui que ce soit!

La bêtise n'est pas d'un côté et l'esprit de l'autre. C'est comme le vice et la vertu ; malin qui les distingue.

Axiome : Le synthétisme est la grande loi de l'ontologie. ${ }^{39}$

$\mathrm{Au}$ contraire le jugement de Bouvard et Pécuchet a toujours une puissance de refus et d'exclusion anti-synthétique. C'est cela savoir et croire : nier, couper, séparer. Tout autre est l'activité artistique. Le paradoxe, c'est que la Critique - telle que la conçoit Flaubert devient une paradoxale puissance d'acceptation : être sûr que tout passe, que le monde est un «flux » comme le suggère le Diable de 1874, c'est cela qui rend possible une forme de détachement que Flaubert appelle la " contemplation ». Dans son échange épistolaire avec Mlle Leroyer de Chantepie qui est torturée de doutes, il combat sa dévotion et les tourments qu'elle lui inflige en vantant les mérites de ce qu'il appelle la « contemplation ardente des choses » :

Je crois que toutes vos douleurs morales viennent surtout de l'habitude où vous êtes de chercher la cause. Il faut tout accepter et se résigner à ne pas conclure. Remarquez que les sciences n'ont fait de progrès que du moment où elles ont mis de côté cette idée de cause. Le Moyen Âge a passé son temps à rechercher ce que c'était que la substance, Dieu, le mouvement, l'infini, et il n'a rien trouvé, parce qu'il était intéressé, égoïste, pratique dans la recherche de la vérité. (Ceci doit être un enseignement pour les individus.) - "Qu'est-ce que ton devoir? L'exigence de chaque jour. » Ceci est un mot de Goethe. Notre devoir est de vivre (noblement, cela va sans dire), mais rien de plus. Or, je ne connais rien de plus noble que la 
contemplation ardente des choses de ce monde. La science deviendra une foi, j'en suis sûr. Mais, pour cela, il faut sortir des vieilles habitudes scolastiques : ne pas faire ces divisions de la forme et du fond, de l'âme et du corps, qui ne mènent à rien ; - il n'y a que des faits et des ensembles dans l'Univers. ${ }^{40}$

Il multiplie les déclarations qui l'engagent à un scepticisme tranquille, et à une activité supérieure qu'il désigne par les mots «science » ou « contemplation »: «Saisirons-nous jamais l'absolu ? Il faut, si l'on veut vivre, renoncer à avoir une idée nette de quoi que ce soit. $»^{41}$; «L'infini, d'ailleurs, submerge toutes nos conceptions et, du moment qu'il est, pourquoi y aurait-il un but à une chose aussi relative que nous? $»^{42}$; «le scepticisme n'aura rien d'amer, car vous serez comme à la comédie de l'humanité et il vous semblera que l'Histoire a passé sur le monde pour vous seule $»^{43} ;$ «La vie est un éternel problème, et l'histoire aussi, et tout. Il s'ajoute sans cesse des chiffres à l'addition. D'une roue qui tourne, comment pouvez-vous compter les rayons ? $»^{44} ;$ «Tâchez de vous cramponner à la science, à la science pure ; aimez les faits pour eux-mêmes. Étudiez les faits comme les naturalistes étudient les mouches. La contemplation peut être pleine de tendresses $»^{45}$. La science telle que la conçoit Flaubert ne ressemble guère à celle que pratiquent Bouvard et Pécuchet qui demandent aux livres des savoirs capables de susciter leur croyance ${ }^{46}$, par leur simplicité, leur évidence et leur efficacité à mater le réel. C'est là une position intéressée - terme flaubertien - qui caractérise le rapport bourgeois au monde. Bouvard et Pécuchet ont le goût des discours et des théories; Flaubert aime dans la science une méthode qui tient compte de la dynamique du monde, de l'infini du réel, de l'ensemble ondoyant des discours.

Le scepticisme, le synthétisme ou la "Bonté » de l'artiste se distinguent de la molle acceptation des bourgeois. Lorsque Flaubert utilise le mot "acceptation", il précise sa spécificité : il évoque «l'acceptation ironique de l'existence, et sa refonte plastique et complète dans l'art $\aleph^{47}$. Bouvard et Pécuchet ont deux travers: ils sont incapables d'acceptation (de bonté, de synthétisme) et encore plus d'ironie. C'est un épouvantable esprit de sérieux qui les ridiculise bien plus que leurs échecs. S'en prendre à la bêtise, c'est le comble de l'Ineptie, qui témoigne encore de leur ridicule esprit de sérieux, c'est une Conclusion sur les conclusions, l'ultime métamorphose du désir de Vérité.

Car Bouvard et Pécuchet souffrent bien de l'illusion qu'il y a une Vérité par rapport à laquelle il existe une Bêtise. D'une discipline à l'autre, ils cherchent un point fixe, une certitude sur laquelle s'appuyer, refusant le temps de l'histoire des sciences qui transforme les connaissances et les représentations, et la multiplicité des points de vue qui relativise toutes les représentations dans le domaine des sciences humaines. Ils refusent surtout la complexité et la nature infinie du réel qui se manifeste même dans ses plus petites parties : ils ne comprennent pas les acides et butent sur la théorie des atomes qui devait permettre de les expliquer (117). Ils manquent d'une part de ce «sens historique » dont Flaubert fait l'éloge dans la Correspondance ${ }^{48}$ et d'autre part du sens de l'infini qui permet d'accepter les mystères du réel. Ils exigent de chaque discipline abordée des certitudes aussi stables que celles des dogmes. Dans le domaine des sciences humaines, ils sont confortés dans leurs illusions par Dumouchel qui ne supporte pas les divergences des historiens : «l'Institut devrait établir une sorte de canon, prescrivant ce qu'il faut croire! » (191).

30 Les contradictions des livres ou les échecs de leurs expériences amènent les deux personnages à abandonner chaque discipline pour une autre, comme Antoine dans $L a$ Tentation lorsqu'il repousse une à une les religions qu'il a pourtant souhaité connaître. Il 
cède au désir de Vérité absolue que lui soufflait Apollonius de Tyane : «Par-dessus toutes les formes, plus loin que la terre, au-delà des cieux, réside le monde des Idées, tout plein du Verbe! D'un bond nous franchirons l'autre espace; et tu saisiras dans son infinité l'Éternel, l'Absolu, l'Être ! $»^{49}$. Bouvard et Pécuchet semblent animés de ce désir prométhéen, et ils espèrent toujours atteindre une chimérique Vérité dans la discipline d'à côté. Le récit est en grande partie structuré par les glissements d'une science à l'autre plus que par l'articulation des événements d'une vie. La Vérité est comme cette Chimère insaisissable qui dans La Tentation de saint Antoine échappe au Sphinx mélancolique qui voudrait la saisir une fois pour toutes et l'arrêter, monter sur son dos, lui qui n'a plus rien à dire et semble avoir perdu le savoir.

Bouvard et Pécuchet demandent aux savoirs de susciter leur foi mais ils exigent cependant de comprendre pour croire comme si tout le monde pouvait être savant de même que tout le monde peut-être croyant, et comme si les sciences donnaient accès à des vérités sans histoire. Selon Flaubert, le roman pourrait avoir pour sous-titre « $\mathrm{Du}$ défaut de méthode dans les sciences $»^{50}$ : en effet, les personnages confondent deux régimes différents du rapport à la vérité. Ainsi ils abandonnent la physiologie qui ne répond pas à leur besoin dogmatique : «On ne sait même pas quelle est la force du cœur. Borelli admet celle qu'il faut pour soulever un poids de cent quatre-vingt mille livres, et Keill l'évalue à huit onces, environ. D'où ils conclurent que la Physiologie est (suivant un vieux mot) le roman de la médecine. N'ayant pu la comprendre, ils n'y croyaient pas. » (127). Leur approche des sciences est métaphysique ce dont témoigne bien le curieux débat entre Vaucorbeil et Pécuchet : «Et une dispute s'engagea sur la nature des fièvres. Pécuchet croyait à leur essence. » (131) Qu'il s'agisse de sciences du vivant ou de sciences humaines, la position est la même et l'abandon d'une thèse ou d'une discipline ressemble toujours à la mort d'un dieu, qui laisse les deux personnages désenchantés. On le voit dans le cas du matérialisme - «Bouvard ne croyait même plus à la matière. » (316) - ou de la littérature : « Bouvard, de moins en moins, croyait à l'esthétique » (220). Incompétents ils ne peuvent comprendre, et ils confondent les limites de leur esprit avec celles de la science. Attachées à expliquer les phénomènes en fonction de connaissances souvent provisoires ou incomplètes, ils interrogent les sciences sur leur capacité à arrêter la recherche scientifique! Bouvard et Pécuchet refusent la profondeur infinie du réel.

Flaubert est fasciné par le temps et l'infini. Son "pessimisme»- mot qu'il emploie lorsqu'il reconnaît son propre penchant dans l'idéalisme de Schopenhauer - et le sentiment de la finitude ne sont pas incompatibles avec des moments de bonheur devant la profusion chatoyante de la vie et son inépuisable flux, dont la Correspondance donne des exemples ${ }^{51}$. Bouvard et Pécuchet sont incapables de ce bonheur, à une exception près. Ils ont lu Cuvier, Geoffroy Saint-Hilaire, Lamarck. Bouvard, un peu égaré, a émis l'hypothèse d'une "matière ondoyante et fugace " (159) qui échappera toujours au savoir. Découragé, il s'endort. Seul et délivré du désir de savoir, Pécuchet se met à regarder véritablement le monde pour une fois. Résumés et discussions cèdent la place à une description poétique qui troue le roman:

Une lisière de mousse bordait un chemin creux, ombragé par des frênes dont les cimes légères tremblaient. Des angéliques, des menthes, des lavandes exhalaient des senteurs chaudes, épicées; l'atmosphère était lourde; et Pécuchet, dans une sorte d'abrutissement, rêvait aux existences innombrables éparses autour de lui, aux insectes qui bourdonnaient, aux sources cachées sous le gazon, à la sève des plantes, aux oiseaux dans leurs nids, au vent, aux nuages, à toute la Nature, sans chercher à découvrir ses mystères, séduit par sa force, perdu dans sa grandeur. (159) 
une brève échappée, par laquelle Flaubert laisse entrevoir une autre attitude possible devant le monde : la contemplation et la rêverie. Il n'est plus alors question de sens mais de force et de grandeur - deux caractéristiques du Beau, selon l'écrivain. Cet épisode ne fait que mieux ressortir le problème de Bouvard et Pécuchet dans le reste du livre : ils ont généralement ce travers qui était déjà celui de Homais et que révèle un lapsus du docteur Larivière, consulté par Mme Homais sur le sang épaissi de son mari par le sommeil de l'après-dîner: "Oh! ce n'est pas le sens qui le gêne " ${ }^{52}$. Comme Homais, Bouvard et Pécuchet ont la passion du sens. Or, dès 1845, Flaubert faisait cette réflexion, suggérant un autre rapport au monde : «Ce qui n’a pas de sens a un sens supérieur à ce qui en a $»^{53}$.

\section{Voir ou savoir}

Toutes les métaphores de l'œuvre d'art ou de l'artiste que l'on peut trouver dans la Correspondance indiquent que le sens n'est pas la finalité de l'œuvre. C'est en cela que l'art pour Flaubert est supérieur à d'autres pratiques humaines (la religion, la philosophie, la politique). Voir ${ }^{54}$ et représenter ${ }^{55}$, devenir cil $^{56}$ et exposer, se faire voir et faire rêver le lecteur ${ }^{57}$, telles sont les formules que l'on trouve sous la plume de Flaubert dans la Correspondance. Et lorsqu'il critique la poésie de Louis Colet, il reproche à la poétesse de ne pas réussir à voir.

Bouvard et Pécuchet sont des anti-artistes : ils sont incapables de voir le réel, ayant toujours devant eux l'écran des discours à valider, avec le désir d'agir sur le monde. Aussi l'une des sources principales du comique et l'un des ressorts du récit, c'est la rébellion du réel que les deux personnages ne perçoivent parfois même pas. Les choux de Pécuchet sont incomestibles mais l'un d'eux le console : « Un, surtout, lui donna des espérances. Il s'épanouissait, montait, finit par être prodigieux, et absolument incomestible. N'importe! Pécuchet fut content de posséder un monstre.» (86) Faisant des conserves, les deux personnages s'applaudissent comme M. Appert pour «avoir fixé les saisons» et ils estiment que de telles découvertes l'emportent "sur les exploits des conquérants.» (111). Ils sont surtout comblés lorsque leurs bouteilles de Chablis explosent (112), manifestant avec éclat la réussite du processus de champagnisation. Flaubert relate les révoltes de la nature et du réel contre les entreprises des deux savants qui veulent les contraindre au nom de la science. Ils imaginent toutes sortes de pratiques pour détourner la force de la nature au profit d'une surproduction bien peu naturelle, mais elle leur échappe par un excès de vie :

La chaleur des meules devint si forte qu'on ne pouvait plus en approcher. Sous les flammes dévorantes la paille se tordait avec des crépitations, les grains de blé vous cinglaient la figure comme des grains de plomb. Puis, la meule s'écroulait par terre en un large brasier, d'où s'envolaient des étincelles ; - et des moires ondulaient sur cette masse rouge, qui offrait dans les alternances de sa couleur, des parties roses comme du vermillon, et d'autres brunes comme du sang caillé. La nuit était venue ; le vent soufflait; des tourbillons de fumée enveloppaient la foule; - une flammèche, de temps à autre, passait sur le ciel noir. (92)

La Nature semble les provoquer: "Bouvard tâcha de conduire les abricotiers. Ils se révoltèrent. Il abattit leurs troncs à ras du sol ; aucun ne repoussa. Les cerisiers, auxquels il avait fait des entailles, produisirent de la gomme. » (96). Le vent jette bas les rames des haricots (86), le dégel fait perdre les artichauts, les orages renversent les épis (86). La 
nature humaine ne leur donne pas plus de satisfaction : « De graves désordres eurent lieu. La fille de basse-cour devint enceinte. Ils prirent des gens mariés ; les enfants pullulèrent, les cousins, les cousines, les oncles, les belles-sœurs. Une horde vivait à leurs dépens [...] » (84). Le comique tient à cette rébellion de toutes choses comme si la Providence s'ingéniait à déjouer leur volonté de maîtrise :

[...] le tonnerre retentit et la pluie tomba, - une pluie lourde et violente. Le vent, par intervalles, secouait toute la surface de l'espalier. Les tuteurs s'abattaient l'un après l'autre - et les malheureuses quenouilles en se balançant entrechoquaient leurs poires.

Pécuchet surpris par l'averse s'était réfugié dans la cahute. Bouvard se tenait dans la cuisine. Ils voyaient tourbillonner devant eux, des éclats de bois, des branches, des ardoises ; - et les femmes de marin qui sur la côte, à dix lieues de là regardaient la mer, n'avaient pas l'œil plus tendu et le cœur plus serré. Puis tout à coup, les supports et les barres des contre-espaliers avec le treillage, s'abattirent sur les plates-bandes. (97)

La réaction des deux horticulteurs positivistes est cocasse: "nous ne sommes guère favorisés!» - et ils se plaignirent «de la Providence et de la Nature» (98). Dans sa Correspondance, Flaubert se moquait volontiers d'un anthropocentrisme similaire :

Ce n'est pas sans un certain plaisir que j'ai contemplé mes espaliers détruits, toutes mes fleurs hachées en morceaux, le potager sens dessus dessous. En contemplant tous ces petits arrangements factices de l'homme que cinq minutes de la nature ont suffi pour bousculer, j'admirais le vrai ordre se rétablissant dans le faux ordre. Ces choses tourmentées par nous, arbres taillés, fleurs qui poussent où elles ne veulent (pas), légumes d'autres pays, ont eu dans cette rebuffade atmosphérique une sorte de revanche. Il y a là un caractère de grande farce qui nous enfonce. Y a-t-il rien de plus bête que des cloches à melon? Aussi ces pauvres cloches à melon en ont vu de belles! Ah! Ah! Cette nature sur le dos de laquelle on monte et qu'on exploite si impitoyablement, qu'on enlaidit avec tant d'aplomb, que l'on méprise par de si beaux discours, à quelles fantaisies peu utilitaires elle s'abandonne quand la tentation lui en prend! Cela est bon. On croit un peu trop généralement que le soleil n'a d'autre but ici-bas que de faire pousser les choux. ${ }^{58}$

Les désastres naturels et les épidémies sont comme des rappels de ce que Flaubert appelle alors « la Règle » : elle fait une place au « Mal - contingent qui n'est peut-être pas le Bien - nécessaire, mais qui est l'être enfin: chose que les hommes voués au néant comprennent peu ${ }^{59}$. Ce que Flaubert appelle ici «l'être », il le nomme ailleurs « infini ». Bouvard et Pécuchet refusent leur finitude, et agissent comme si l'homme pouvait être le centre du monde, et que celui-ci n'attendait que ses progrès pour se soumettre à sa raison.

Contrairement à ce point de vue scientiste hérité de vieux préjugés chrétiens, Flaubert prend plaisir à imaginer la petitesse de l'homme enveloppé dans le grand infini. Il se moque des « esprits présomptueux et enthousiastes » qui veulent prendre « la dimension de l'infini $»^{60}$. La nature et l'Histoire humaines ne sont pas moins irreprésentables dans leur totalité que l'idée de Dieu pour Spinoza, seul philosophe à avoir échappé à sa critique. Flaubert n'est certes pas un philosophe - à partir de la seconde moitié des années 1840 il dit même dans sa Correspondance toute sa méfiance à l'égard des philosophies bien qu'il continue à en lire - et il serait difficile de définir sa philosophie autrement que par un certain nombre de refus et d'idées assez larges: une critique de tous les systèmes au nom de l'infini, le sentiment d'une finitude humaine débordée de toutes parts, la conscience sereine de la mort, le refus des dualismes (en particulier de la distinction entre le corps et l'esprit, du matérialisme et du spiritualisme). Toutefois, on 
sait qu'il a lu l'Éthique dans les années 1840, peut-être sur les conseils d'Alfred Le Poittevin qui l'admirait au point de le lire encore en 1848, dans les derniers jours de sa vie. Spinoza a sans doute aidé Flaubert à concevoir l'idée d'unité et d'infini. En 1872, il débute les grandes lectures pour Bouvard et Pécuchet et souffre en lisant Kant et Hegel. Il se reporte alors à son Spinoza, qu'il « repasse » avec plaisir ${ }^{61}$. Les autres philosophies le lui rendent encore plus précieux : «[...] quand je sors de leur compagnie je tombe avec voracité sur mon vieux et trois fois grand Spinoza! Quel génie! Quelle œuvre que l'Éthique! »62. Significativement Spinoza échappe au jeu de massacre dans le chapitre VIII de Bouvard et Pécuchet : «L'Éthique les effraya avec ses axiomes, ses corollaires. » (302) Leur résumé de cette philosophie s'achève sur un anti-dualisme bien flaubertien mais formulé de manière cocasse par un enveloppement de la matière dans la pensée et vice versa qui les laisse perplexes, mais qui déjoue le rapport de causalité typique d'une logique discursive :

Ainsi notre monde n'est qu'un point dans l'ensemble des choses - et l'univers impénétrable à notre connaissance, une portion d'une infinité d'univers émettant près du nôtre des modifications infinies. L'Étendue enveloppe notre univers, mais est enveloppée par Dieu, qui contient dans sa pensée tous les univers possibles, et sa pensée elle-même est enveloppée dans sa substance.

Il leur semblait être en ballon, la nuit, par un froid glacial, emportés d'une course sans fin, vers un abîme sans fond, - et sans rien autour d'eux que l'insaisissable, l'immobile, l'Éternel. C'était trop fort. Ils y renoncèrent. (303).

L'enveloppement anti-dualiste est aux antipodes du rationalisme de Bouvard et Pécuchet qui ont besoin d'oppositions et de conclusions. Flaubert indique en creux et en abyme dans le texte - grâce à l'abandon de cette lecture - la pensée antilogique qui préside à la construction de ce roman sceptique, écrit à la gloire de l'infini. Roman sur le caractère problématique du réel, sur les limites de l'homme, cette œuvre est une réflexion expérimentale sur la question de la connaissance. La fiction a une valeur exploratoire et interrogative. Flaubert imagine une autre conception du cognitif et réinvente une nouvelle forme de « roman philosophique $»^{63}$.

\section{Conclusion}

41 Encyclopédie en farce, revue de toutes les idées d'une époque, c'est ainsi que Flaubert désigne cette œuvre étrange qu'est Bouvard et Pécuchet, le pendant de La Tentation de saint Antoine. Après les croyances religieuses, ce sont les croyances scientifiques qui sont mises en fiction et en question dans un débat où chacun des deux personnages joue par rapport à l'autre un rôle critique, la position du croyant et du Diable ne cessant de tourner. Cette structure critique s'en prend au dogmatisme ${ }^{64}$, au rêve scientiste d'une conversion de la science en nouvelle foi. L'œuvre met en cause l'illusion d'une adéquation entre les représentations et les choses, et l'illusion qu'il serait possible d'atteindre une objectivité par-delà les représentations. Cet espoir est celui de Bouvard et Pécuchet lorsqu'ils projettent d'écrire un livre d'histoire en "reflétant la vérité tout entière " (192) et en évitant tout point de vue limitatif. Cette histoire ne prend évidemment pas forme. La fiction flaubertienne ne porte donc pas sur la recherche scientifique et la validité de ses procédures mais sur l'inadéquation d'une approche. Bouvard et Pécuchet attendent des livres qu'ils donnent sens et stabilité à l'univers, qu'ils le fixent et l'arrêtent, qu'ils en rendent compte complètement. Ils leur demandent des formules simples pour agir sur le réel. Leur comportement à l'égard du réel est religieux et magique : les bons mots, les bons gestes doivent produire des miracles, des monstres, des guérisons. Comme Emma 
Bovary, Bouvard et Pécuchet croient sans distance aux livres et aux fictions qu'ils s'inventent à partir de quelques-uns de leurs énoncés. Ils partagent avec elle une sorte de donquichottisme, le désir de donner vie aux mots. Bouvard et Pécuchet peut se lire comme une anti-épopée à la manière de Don Quichotte : le roman confronte les livres et le réel et les expériences scientifiques sont comme des exploits qui tournent mal et d'où le réel sort toujours victorieux, manifestant ainsi son irréductible différence par rapport aux théories. À une époque où le scientisme ambiant laisse encore espérer à une relève de la religion par la science, Flaubert voit l'irréductibilité de l'une à l'autre et la nécessité d'une critique - sinon d'une archéologie - des savoirs. Convaincu qu'il n'y a que « des manières de voir " $"$, Flaubert se fait le romancier des représentations. Dans Bouvard et Pécuchet la fiction ne crée pas d'illusion réaliste - elle réduit au minimum les descriptions des personnages et la psychologie ainsi que les péripéties romanesques qui pourraient créer l'illusion d'une vie - mais au contraire elle fonctionne comme une machine à surexposer plus qu'à détruire - toutes les illusions. Même si bien des incertitudes demeurent quant au second volume, on peut se demander si la Copie des deux personnages ne devait pas constituer une forme de renoncement à la Vérité, une sorte de conversion à «l'acceptation ironique» de toutes les représentations, tenant à distance le jugement grâce à la pratique hospitalière de la copie. Flaubert imaginait peut-être un équivalent moderne de l'extase de saint Antoine devant la profusion infinie des formes imaginaires et des formes vivantes à la fin de La Tentation de 1874, lorsque l'ermite désire non plus savoir ce que pense la matière comme dans le texte de 1849 mais seulement être la matière et vibrer en elle. De la vision extatique à la copie, de la contemplation du saint à la collection des copistes, un point commun : le renoncement au savoir et à la croyance. Toutefois, les deux œuvres font encore un tour de plus comme si la critique devait toujours être encore ressaisie par la critique ${ }^{66}:$ la vision de la vie et le désir d'être matière interviennent dans une dernière hallucination de saint Antoine; la copie de Bouvard et Pécuchet devait être - semblerait-il selon les notes manuscrites de Flaubert interrompue par quelques réflexions sur les difficultés rencontrées par les personnages pour éviter un classement et le retour subreptice du jugement ${ }^{67}$.

\section{NOTES}

1. Voir Nicole Dhombres, Les Savants en révolution, Paris, Calmann-Lévy, s.d. ; Lise Andries (dir.), La construction des savoirs. XVIII ${ }^{e}$-XIX ${ }^{e}$ siècles, Presses universitaires de Lyon, 2009.

2. Voir par exemple la lettre à Mlle Leroyer de Chantepie du 18 février 1859 ; Corr., III, p. 17. La Correspondance est citée dans l'édition de Jean Bruneau et Yvan Leclerc, Gallimard, coll. «Bibliothèque de la Pléiade ", 1973-2007.

3. Lettre à L. Colet du 24 avril 1852 ; Corr., II, p. 76.

4. Lettre à L. Colet du 24 avril 1852 ; Corr., p. 78.

5. Lettre à L. Colet du 31 mars 1853 ; Corr., II, p. 295.

6. Lettre à L. Colet du 6 avril 1853 ; Corr., II, p. 298.

7. Carnet 2, fo 5, daté de 1859 par Pierre-Marc de Biasi, Carnets de travail, Balland, 1988, p. 213. Les soufflets indiquent une addition. 
8. Lettre à L. Colet du 27 mars 1852 ; Corr., II, p. 62.

9. Lettre à L. Bouilhet du 4 septembre 1850 ; Corr., I, 679.

10. Cours de philosophie positive, Paris, Rouen Frères, 1830, I, p. 14.

11. Voir L'Avenir de la science de Renan (1848, publié en 1890).

12. Lettre à L. Bouilhet du 4 septembre 1850 ; op. cit. C'est Flaubert qui souligne.

13. Science et nature. Essai de philosophie et de science naturelle, traduit de l'allemand par Augustin Delondre, Paris, Germer Baillière, 1966, I, chapitre III. Une note du dossier de Bouvard et Pécuchet (manuscrit g 226, vol. 4, $\mathrm{f}^{\circ} 55 \mathrm{r}^{\circ}$ ) évoque cette lecture.

14. Lettre à Louise Colet du 26 mai 1853 ; Corr., II, p. 334.

15. Lettre à Louise Colet, 7 juillet 1853 ; Corr., II, p. 378.

16. Propos recueillis par Auguste Sabatier et cité dans son article du 3 avril 1881 dans le Journal de Genève.

17. «Je vais me mettre, cet été, à un autre livre du même tonneau [...] », annonce-t-il à G. Sand le $1^{\mathrm{er}}$ mai 1874.

18. «Fiction et transgression épistémologique : le mythe de l'origine dans La Tentation de saint Antoine de Flaubert », Romanic Review, janvier 1997, p. 131-144.

19. Une édition de la première Tentation de saint Antoine (posthume) est accessible dans la coll. de l'Intégrale aux Éditions du Seuil (1964). Les passages cités dans cet article ont été établis à partir du manuscrit de l'œuvre conservé à la BnF (cote N.a.fr. 23664).

20. Lettre à Feydeau de novembre 1857 ; Corr., II, p. 783.

21. Lettre à Louise Colet du 27 février 1847 ; Corr., I, p. 441.

22. Une édition de la première Tentation de saint Antoine (posthume) est accessible dans la coll. de l'Intégrale aux Éditions du Seuil. Les passages cités dans cet article ont été établis à partir du manuscrit de l'œuvre conservé à la BnF (cote N.a.fr. 23664).

23. Renan l'écrit en 1848 et ne le publie qu'en 1890 comme un témoignage sur cette époque. Il affirme alors avoir pris ses distances par rapport à certaines idées comme celle d'un remplacement de la Religion par une Science toute-puissante et triomphante.

24. Lettre à L. Colet du 27 mars 1853 ; Corr., II, p. 283.

25. Madame Bovary, édition établie par J. Neefs, Le Livre de Poche classique, II, chap. 11, p. 283.

26. Voir G. Séginger, Naissance et métamorphose d'un écrivain. Les Tentations de saint Antoine, Champion, 1997.

27. La Tentation de saint Antoine, édition de Claudine Gothot-Mesch, Gallimard, coll. « Folio », 1983, p. 206.

28. Ibid., p. 215.

29. Ses lectures l'enthousiasment (voir la lettre à Maxime Du Camp d'avril 1846).

30. Flaubert en a découvert la théorie dans l'Introduction à l'histoire du bouddhisme indien (1844) d'Eugène Burnouf.

31. Lettre à Mme Roger des Genettes du 11 juin 1879 ; Corr., V, p. 659.

32. "La Forme est peut-être une erreur de tes sens, la Substance une imagination de ta pensée. » (op. cit., p. 214).

33. Ibid., p. 215.

34. Lettre à Mlle Leroyer de Chantepie du 6 juin 1857 ; Corr., II, p. 731.

35. Ainsi en 1874, lorsque Sylvestre de Sacy - un savant spécialiste des langues orientales et secrétaire perpétuel de l'Académie des Inscriptions - lui avoue ne jamais avoir lu ni Lucrèce ni Pétrone, il est scandalisé : «Ô France ! Bien que ce soit notre pays, c'est un triste pays, avouonsle! Je me sens submergé par le flot de bêtise qui le couvre, par l'inondation de crétinisme sous laquelle peu à peu il disparaît. Et j'éprouve la terreur qu'avaient les contemporains de Noé, quand ils voyaient la mer monter toujours. Les plus grands bénisseurs, tel que le père Hugo, commencent eux-mêmes à douter. » (17 juin, Corr., IV, p. 814).

36. Lettre à Mme Roger des Genettes du 24 janvier 1880 ; Corr., V, p. 796. 
37. Jules qu'il oppose au bourgeois Henry.

38. Lettre à Alfred Le Poittevin du 16 septembre 1845 ; Corr., I, p. 252.

39. Lettre à L. Bouilhet, $1 / 8 / 55$; Corr., II, p. 586.

40. Lettre du 18 décembre 1859. Jouant sur le titre du livre de piété bien connu d'Ignace de Loyola, il lui écrivait le 18 mai 1857 : «Il y a un sentiment ou plutôt une habitude dont vous me semblez manquer, à savoir l'amour de la contemplation. Prenez la vie, les passions et vous-même comme un sujet à exercices intellectuels » (Corr., II, p. 718).

41. Lettre à Mlle Leroyer de Chantepie du 18 mai 1857 ; Corr., II, p. 716.

42. Lettre à Mlle Leroyer de Chantepie du 6 juin 1857 ; op. cit.

43. Lettre à Mlle Leroyer de Chantepie du 18 mai 1857 ; Corr., II, p. 718.

44. Ibid.

45. Lettre du 8 septembre 1860 ; Corr., III, p. 110.

46. Sur le rapport du savoir et de la croyance, voir aussi Stéphanie Dord-Crouslé, Bouvard et Pécuchet de Flaubert, une encyclopédie en farce, Belin, 2000, chap. V.

47. Lettre à Louise Colet du 23 janvier 1854 (Corr., II, p. 514). Flaubert fera l'éloge dans la prose de Tourgueneff d'une telle manière de voir: "Quel mélange d'attendrissement, d'ironie, d'observation et de couleur!» (lettre du 12 février 1866 ; Corr., III, 310).

48. Lettre à Mlle Leroyer de Chantepie du 18 février 1859 ; Corr., II, p. 16.

49. La Tentation de saint Antoine, op. cit., p. 159.

50. Lettre à Gertrude Tennant du 16 décembre 1879 ; Corr., V, p. 767.

51. Voir par exemple la lettre à Louis Bouilhet du 24 août 1856 ou la lettre à Caroline du 21 janvier 1877 (Corr., V, p. 174).

52. Madame Bovary, op. cit., p. 468.

53. Lettre à Alfred Le Poittevin de 1845 ; Corr., I, p. 252.

54. Lettre à Louise Colet 1-2 octobre 1852 ; Corr., p. 165.

55. Lettre à Louise Colet du 13 septembre 1852 ; Corr., II, p. 157.

56. Lettre à Louise Colet du 27 septembre 1846 (Corr., I, p. 364) et du 22 avril 1853 (Corr., II, p. 313).

57. Lettre à Louise Colet du 26 août 1853 ; Corr., II, p. 417.

58. Lettre à Louise Colet du 12 juillet 1853 ; Corr., II, p. 381.

59. Ibid.

60. Lettre à Mlle Leroyer de Chantepie du 18 mai 1857.

61. Lettre à Georges Sand du 26 février 1872 ; Corr., IV, p. 486.

62. Lettre à George Sand du 31 mars 1872 ; Corr., IV, p. 505.

63. Flaubert désigne ainsi son roman à Auguste Sabatier (op. cit.).

64. Sur le dogmatisme et le scepticisme, sur l'influence possible de Spencer voir aussi Yvan Leclerc, La Spirale et le monument, SEDES, 1988, p. 90-92.

65. Lettre à Léon Hennique du 3 février 1880 ; Corr., V, p. 811.

66. Sur ce point voir aussi G. Séginger, «L'art critique », Flaubert. Une éthique de l'art pur, SEDES, 2000, réédition Minard, Caen, 2010, p. 191-214.

67. Voir par exemple le $\mathrm{f}^{\circ} 67 \mathrm{r}^{\circ}$ du manuscrit gg 10, cité en annexe de l'édition de référence (p. 442). 
INDEX

Mots-clés : croyance, savoir, science, positivisme, méta-science, Tentation de saint Antoine (La)

\section{AUTEUR}

GISĖLE SÉGINGER

LISAA - EA 4120 - Université Paris-Est Marne-la-Vallée 